\title{
Relación de los pequeños mamíferos terrestres (Rodentia y Didelphimorphia) con la estructura de la vegetación en el Bosque Atlántico Interior - un análisis multivariado
}

\author{
María Belén Barreto Cáceres ${ }^{1}$ y Robert D. Owen ${ }^{2 *}$ \\ ${ }^{1}$ Facultad de Ciencias Exactas y Naturales, Departamento de Biología, Universidad Nacional de Asunción. San Lorenzo, Paraguay. \\ Email: mariabelenbio@gmail.com (MBBC). \\ ${ }^{2}$ Centro para el Desarrollo de la Investigación Científica, Asunción, Paraguay, and Department of Biological Sciences, Texas Tech \\ University, Lubbock. Texas, U.S.A. Email: rowen@pla.net.py (RDO). \\ * Corresponding author
}

The ecological importance of small mammals includes their role as dispersers of seeds, spores and propagules of plants, fungi and lichens, decomposers of organic matter and controllers of invertebrates as well as prey of a great number of vertebrates. The objective of this research was to determine the relationship between non-volant small mammals (Rodentia and Didelphimorphia) to vegetation structure and the extent of degradation of the forest in an area near the western boundary of the Upper Parana Atlantic Forest, and to evaluate seasonal and interannual variation in species' abundances. The study was conducted in the Mbaracayú Forest Natural Reserve, Paraguay. Mark-capture methodology was employed in three sites with different degrees of degradation, in two seasons of the year (dry and rainy), in 2015 and 2016. A multivariate analysis was performed with ANOVA and MANOVA, evaluating degree of habitat disturbance and annual and seasonal variation as independent variables and species abundances as dependent variables. Sixteen small-mammal species were recorded, with 1,161 captures of 598 individuals and 563 recaptures throughout the sampling. Twelve species were of the Order Rodentia (all of the family Cricetidae, subfamily Sigmodontinae), and four of the Order Didelphimorphia (family Didelphidae). Variables that were significant for the most abundant species (Akodon montensis) were interannual variation and habitat degradation. The most common didelphid species in the study was Gracilinanus agilis, which appears well-adapted to different degrees of habitat degradation, given its occurrence in all sites. Plant communities, including their composition and degree of habitat disturbance, are important for small-mammal communities which are interacting in the different habitats of the forest. In this study we observed that Akodon montensis was most abundant in the habitat of moderate disturbance in the dry season (June). This habitat was characterized by large trees with grasses predominant in the understory. Hylaeamys megacephalus was most abundant in the dry season (June) and less so in the rainy season (November). Oligoryzomys nigripes is a habitat generalist and is less specialized in food preference. Abundance of the didelphid species Gracilinanus agilis was quite variable among sampling periods, but the species was present in all three levels of habitat degradation. The various species (especially the most abundant) exhibited different responses to habitat degradation and to interannual and seasonal variations.

La importancia ecológica de los micromamíferos radica en el rol que desempeñan en los bosques, donde actúan como dispersores de semillas, esporas y propágulos de plantas, hongos y líquenes, descomponedores de materia orgánica, controladores de invertebrados y a la vez, como presas de gran número de vertebrados. El objetivo de esta investigación fue determinar la relación de los micromamíferos no voladores (Rodentia y Didelphimorphia) con la estructura de la vegetación y el nivel de degradación del bosque en una zona del límite occidental del Bosque Atlántico del Alto Paraná y evaluar variación estacional e interanual en las abundancias de estas especies. Se llevó a cabo en la Reserva Natural del Bosque Mbaracayú, Paraguay. La metodología utilizada fue de captura marca recaptura, en tres sitios con diferentes niveles de degradación, en dos épocas del año (seca y lluviosa), en el 2015 y 2016. Se realizó un análisis multivariado con ANOVA y MANOVA considerando el año, época del año y parcela como variables independientes y las abundancias de especies como variables dependientes. El total de especies registradas fue de 16, con 1,161 capturas de 598 individuos, con 563 recapturas. Doce especies fueron del orden Rodentia (todos de la familia Cricetidae, subfamilia Sigmodontinae) y cuatro del orden Didelphimorphia (familia Didelphidae). Las variables que resultaron significativa para la especie más abundante, Akodon montensis, fueron el año y el hábitat (nivel de degradación). La especie de didélfido más común en todo el estudio fue Gracilinanus agilis, que podría estar adaptado a los diferentes niveles de degradación del hábitat, dada su ocurrencia en todos los sitios. Las comunidades vegetales son principalmente importantes para las comunidades de micromamíferos que se encuentran interaccionando en los diferentes hábitats del bosque. En este estudio se observó que Akodon montensis fue más abundante en el hábitat de degradación media en la época seca (junio). En este hábitat estaban presentes comunidades vegetales de árboles de gran porte con predominio de gramíneas en el sotobosque. La población de Hylaeamys megacephalus disminuyó en la época lluviosa, observándose una mayor abundancia en la época seca. Oligoryzomys nigripes podría ser generalista en lo referente al hábitat por ser una de las especies menos especialistas en cuanto a la alimentación. Gracilinanus agilis fluctuó durante todos los periodos de muestreos en las diferentes estaciones del año y estuvo asociado a los tres niveles de degradación. Las asociaciones entre comunidades de micromamíferos y comunidades vegetales indicarían preferencia hacia los diferentes tipos de microhábitats por parte de los micromamíferos. Las distintas especies (sobre todo las más abundantes) responden de diferentes maneras a las modificaciones del hábitat y a lo largo del ciclo anual y en las diferentes épocas debido a sus preferencias u ocurrencia en el hábitat.

Keywords: analysis of variance; canonical coordinates analysis; cluster analysis; Didelphidae habitat association: Reserva Natural del Bosque Mbaracayú; Sigmodontinae.

(c) 2019 Asociación Mexicana de Mastozoología, www.mastozoologiamexicana.org 


\section{Introducción}

Los micromamíferos pueden llegar a ser indicadores del estado de los bosques, dado que la heterogeneidad del espacio en que se encuentran promueve la diversificación de sus nichos espaciales, además de ser dispersores de semillas, esporas y propágulos de plantas, hongos y líquenes, descomponedores de materia orgánica, controladores de los invertebrados y a su vez, presas de un gran número de vertebrados (Aragón et al. 2009).

En el Neotrópico, los roedores sigmodontinos constituyen una parte importante de la riqueza de las comunidades faunísticas (Emmons 1999; Patton et al. 2015). Sin embargo, muchas preguntas sobre selección de hábitat dentro de este grupo siguen sin respuesta. Probablemente, las respuestas a estas preguntas permitirían una mejor comprensión de la composición y la dinámica de las comunidades de roedores a una escala local (es decir, la escala a la que un roedor experimenta su entorno).

En las últimas dos décadas, numerosos estudios han investigado las asociaciones de hábitats de roedores Sigmodontinos en todo el Neotrópico. Schnell et al. (2010) evaluaron las preferencias de hábitat de Sigmodon mascotensis en una zona costera seca del occidente de México, y Poindexter et al. (2012) evaluaron cuatro especies comunes de roedores en la misma área. Delciellos et al. (2016) informaron que la estructura del hábitat era un determinante importante de ensamblajes de pequeños mamíferos en fragmentos de Mata Atlántica en el estado de Río de Janeiro, Brasil. Además, se han reportado distintas asociaciones de hábitats para una variedad de especies de sigmodontinos en marismas de agua dulce (Bonaventura et al. 2003), remanentes de Bosques Atlánticos secundarios (Püttker et al. 2008), Bosque Atlántico del sur (Melo et al., 2011, 2013), y la región pampeana en el sur de Brasil (Sponchiado et al. 2012). Owen et al. (2010) encontraron asociaciones de hábitats distintas para Akodon montensis en una región del Bosque Atlántico Occidental. Polop et al. (2014) también informaron sobre asociaciones de hábitat para tres especies de Sigmodontinae, Oligoryzomys longicaudatus, Abrothrix hirta (reportado como A. longipilis) y A. olivacea, en una región del sur de los Andes.

Las comunidades de micromamíferos no voladores presentes en áreas boscosas del Bosque Atlántico Interior, al estar relacionadas con las comunidades vegetales, permiten inferir acerca del nivel de degradación del hábitat, ya que responderían de distintas maneras a los cambios que ocurren en el bosque al ser sometidos a una comparación entre sitios, épocas y años. Los niveles de degradación de los bosques afectan la composición de las especies de micromamíferos no voladores tanto a escala temporal como espacial. Se producen comúnmente fluctuaciones irregulares en el número de individuos debido a variaciones climáticas, tales como tiempo particularmente frío, caliente o seco, o también por catástrofes naturales como fuegos o tormentas, y estos factores son independientes de la densidad (Hickman et al. 2002). La explosión demográfica en las poblacio- nes de roedores puede estar ligada a factores ambientales como el fenómeno de El Niño, lo que en países de América del Sur se pudo observar en los últimos años (Jaksic y Lima 2003, Dirección Nacional de Aeronáutica Civil 2016).

En las zonas limítrofes del Bosque Atlántico del Alto Paraná esporádicamente aparecen algunas especies de pequeños mamíferos terrestres encontrados generalmente en bosques o vegetación secundaria y en esas zonas marginales de las ecorregiones la fauna característica de cada ecorregión puede llegar a ser sensible a cambios de su ambiente biótico o abiótico (Owen 2013).

En este trabajo se realizaron evaluaciones de la dinámica de la comunidad de micromamíferos y su asociación con la estructura vegetal en dos épocas del año (seca y lluviosa), a lo largo de un periodo de dos años, utilizando métodos de captura-marca-recaptura en tres parcelas distintas, establecidas de acuerdo a las comunidades vegetales y a los distintos niveles de degradación.

\section{Material y Métodos}

Sitio del estudio. El Bosque Atlántico del Alto Paraná (BAAPA) abarca una superficie de $483.800 \mathrm{~km}^{2}$ y representa uno de los Biodiversity Hotspots del planeta, es decir uno de los sitios claves para la conservación por la diversidad que representa (Myers et al. 2000). Se caracteriza por estar predominantemente conformado por bosque subtropical semicaducifolio húmedo a subhúmedo (Dinerstein et al. 1995; Cartes 2006). Actualmente, los bosques de esta ecorregión existen en fragmentos remanentes que en conjunto totalizan sólo el 7 \% de su extensión original (Cartes 2006).

El área de estudio comprende la Reserva Natural del Bosque Mbaracayú (RNBM), que cuenta con 64.405 hectáreas correspondiente al área núcleo de la Reserva de Biosfera del Bosque Mbaracayú (Owen et al. 2019; Owen y Smith 2019). Se localiza al noreste de la región Oriental del Paraguay, entre los $24^{\circ} 00^{\prime}$ y $24^{\circ} 15^{\prime}$ de latitud Sur, y $55^{\circ} 00^{\prime}$ y $55^{\circ}$ $32^{\prime}$ de longitud Oeste, en el Departamento de Canindeyú (Fundación Moisés Bertoni/Banco Mundial 2005). Toda el área en que se encuentra la RNBM está ubicada en la cuenca del Río Jejuí, un tributario importante del Río Paraguay. La RNBM representa uno de los más grandes fragmentos de bosques que se encuentran protegidos en Paraguay lo que significa tener a disposición una de las pocas oportunidades para estudiar comunidades de roedores en sus hábitats (Eastwood et al. 2018).

El clima se caracteriza por presentar una temperatura media anual de $22{ }^{\circ} \mathrm{C}$ y precipitaciones medias de 2,000 $\mathrm{mm} / \mathrm{año}$, siendo la temporada de lluvia durante los meses de octubre a marzo (primavera-verano). La época seca y fría se da entre los meses de junio y octubre (invierno-primavera). Sin embargo, durante las épocas de El Niño ocurren incrementos de las precipitaciones (Di Bitetti et al. 2003).

Estructura de la vegetación y nivel de degradación. Las tres parcelas de muestreo se evaluaron basándose en una serie extensa de características estructurales de la vegetación, 
medidas en cada una de las estaciones de trampa en cada parcela. Las medias de estas medidas de vegetación se utilizaron como medidas generales para cada parcela. Estos se estandarizaron a una media de cero y una desviación estándar de uno, para mitigar los efectos del tamaño del carácter. Los eigenvectores se extrajeron de la matriz de correlaciones de caracteres estandarizados y se evaluaron mediante el análisis de componentes principales (PCA; Sneath y Sokal 1973). Luego de los análisis preliminares, se incluyeron seis caracteres en el PCA final, como descriptores importantes de la calidad del bosque y el hábitat de roedores: árboles caídos cercanos (AC), distancia al árbol más cercano (DAC), porcentaje de cobertura de hierbas $(\mathrm{PCH})$, restos de madera en el suelo (MM), árbol de naranjo (AN), altura máxima del dosel (AMD). Se consideró que los niveles de degradación estaban asociados positivamente con los dos primeros componentes principales, que en conjunto representaban el $95 \%$ de la varianza. Por lo tanto, un hábitat de bosque más degradado se asocia con las hierbas, los troncos (árboles caídos) y los naranjos (Citrus aurantium, una especie introducida que se ha aclimatado en bosques perturbados). El hábitat de bosque menos degradado se asocia con más madera muerta (ramas caídas, no árboles caídos), un dosel más alto y una menor distancia al árbol más cercano. De los datos de vegetación se analizaron los valores promedios para cada parcela y en cada época de muestreo del año 2015 (Tabla 1). En las dos épocas de muestreo (seca y lluviosa) realizadas, se consideraron las mismas características de la vegetación.

Se designaron las tres parcelas de acuerdo a la calidad del hábitat definida en términos de degradación: Parcela $B$ (degradación baja, con su centro a las $24.141^{\circ} \mathrm{S}, 55.366^{\circ}$ W), A (degradación media, $24.123^{\circ} \mathrm{S}, 55.505^{\circ} \mathrm{W}$ ) y G (degradación alta, $24.131^{\circ} \mathrm{S}, 55.537^{\circ} \mathrm{W}$ ), distantes mínimos a 3.3 $\mathrm{km}$ una de otra. Los nombres y designaciones de las parcelas son consistentes con otras publicaciones en preparación o en revisión basadas en datos desarrollados en este proyecto.

Métodos de muestreo. El tamaño de cada parcela fue de $12 \times 12=144$ estaciones. Las estaciones en cada parcela estuvieron separadas unos 10 metros entre sí. En cada estación se colocaron dos trampas Sherman (7.5 x 9.0 x $23.0 \mathrm{~cm}$; H. B. Sherman Traps, Tallahassee, Florida) en el suelo, y otra a 1-3 m por encima del suelo (lianas o ramas de árboles). En la primera sesión de muestreo (periodo de junio/julio de 2015) se utilizaron una trampa a nivel del suelo y una trampa arriba, aumentando en los siguientes muestreos a una trampa más por estación a fin de aumentar el esfuerzo de muestreo. Las trampas fueron cebadas con una mezcla de avena y mantequilla de maní y revisadas cada mañana.

Se utilizó el método de captura-marca-recaptura. Los muestreos se realizaron en los meses de junio/julio (época seca) y noviembre (época lluviosa) de los años 2015 y 2016. Cada muestreo abarcaba cinco noches en cada parcela. El procedimiento consistió en identificar al animal capturado por medio de una etiqueta PIT (Passive Integrated Transponder, Biomark Inc., Boise, Idaho) de $8.4 \mathrm{~mm}$ de largo implantada por vía subcutánea entre los hombros con una jeringa. Luego de la recaptura estos animales podían ser identificados por medio de un lector electrónico de mano.

Se extrajeron muestras de tejido ( 1 a $2 \mathrm{~mm}$ de la punta de la cola) para su identificación posterior por ADN si fuera necesario, además de la preparación de espécimen de cráneo y fluido, de los especímenes encontrados muertos en trampa, para corroborar las identificaciones hechas en campo. Los especímenes colectados y preparados están albergados en la colección de mamíferos del segundo autor. Se utilizó para la nomenclatura taxonómica y claves de identificación de Patton et al. (2015) para roedores sigmodontinos, y de Gardner (2008) y Voss y Jansa (2009) para marsupiales.

Se tomaron datos de cada individuo capturado como el código de PIT, fecha, parcela, número de estación de trampeo, lugar de colocación de la trampa (suelo, encima del suelo), identidad específica del individuo, sexo, clase de edad, condición reproductiva, peso y la salud en general. Los procedimientos con animales en este estudio se llevaron a cabo bajo los Permisos de Colecta Científica No. 011/2014, 132/2015 y 269/2016 (Secretaría del Ambiente, actualmente el Ministerio de Ambiente y Desarrollo Sostenible, Paraguay), y se siguieron las pautas del Comité de Cuidado y Uso de Animales del Sociedad Americana de Mastozoólogos para el uso de mamíferos silvestres en investigación y educación (Sikes et al. 2011, 2016). Todos los procedimientos con animales fueron aprobados (Aprobación No. 14024-03) por el Comité Institucional de Cuidado y Uso Animal de la Universidad de Texas Tech (IACUC), que sigue la $8^{a}$ Edición de la Guía para el Cuidado y Uso de Animales de Laboratorio (Guía), NRC 2011.

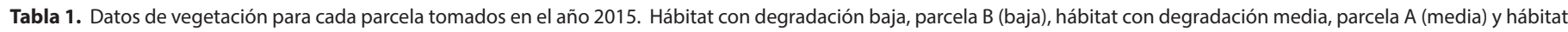
con degradación alta, parcela G (alta).

\begin{tabular}{|c|c|c|c|c|c|c|}
\hline \multirow[b]{2}{*}{ Características de la vegetación } & \multicolumn{2}{|c|}{ Parcela B (baja) } & \multicolumn{2}{|c|}{ Parcela A (media) } & \multicolumn{2}{|c|}{ Parcela G (alta) } \\
\hline & Junio & Noviembre & Junio & Noviembre & Junio & Noviembre \\
\hline Madera muerta en el suelo (\%) & $11.0(12.3)$ & $8.0(15.3)$ & $7.5(11.0)$ & $5.1(9.1)$ & $11.1(13.3)$ & $5.5(9.8)$ \\
\hline Altura máxima del dosel (m) & $16.0(5.1)$ & $14.6(5.2)$ & $15.6(4.8)$ & $14.3(5.0)$ & $15.6(3.0)$ & $13.8(3.3)$ \\
\hline Distancia a los árboles más cercanos (m) & $4.6(1.4)$ & $4.6(1.4)$ & $4.8(1.6)$ & $4.8(1.7)$ & $4.2(1.4)$ & $4.2(1.4)$ \\
\hline Porcentaje de cobertura de hierbas (\%) & $19.1(10.0)$ & $22.3(7.8)$ & $17.5(10.4)$ & $18.8(10.0)$ & $4.9(4.1)$ & $27.4(13.0)$ \\
\hline Árboles caídos (por unidad) & $0.6(0.5)$ & $0.6(0.5)$ & $0.6(0.5)$ & $0.6(0.5)$ & $0.1(0.3)$ & $0.1(0.3)$ \\
\hline Arboles de naranjo (por unidad) & $0.0(0)$ & $0.0(0)$ & $0.3(0.4)$ & $0.3(0.4)$ & $0.6(0.5)$ & $0.2(0.4)$ \\
\hline
\end{tabular}


La colección de mamíferos donde los vouchers están depositados cuenta con Habilitación №. 004/2015 (Secretaria del Ambiente, Paraguay).

Análisis de datos. Se utilizó el Número Mínimo de Conocidos Vivos (Minimum Number Known Alive: MNKA), que es el número total de individuos de una especie encontrado en una parcela durante una sesión de muestreo. Para probar si los datos seguían una distribución normal se realizó la prueba de normalidad de Shapiro-Wilks, para los datos de los dos años. Se rechazó la hipótesis nula que afirma que el conjunto de datos sigue una distribución normal para el 2015 y para el año 2016. Se transformó los datos por medio de Ln (logaritmo natural) para llegar a una distribución normal.

Se utilizó el Análisis de Correspondencia Canónica (CCA) para evaluar la relación especie-ambiente, teniendo en cuenta un conjunto común en donde se asume las respuestas de las especies. Este método se centra principalmente en las relaciones entre especies y mediciones de las variables ambientales, obteniendo una interpretación brindada por los ejes de ordenación. Ha sido desarrollado para estudios de ecología en donde se relaciona la abundancia de especies con las variables ambientales (Ter Braak 1986).

Para evaluar la similitud de las tres parcelas durante los cuatro periodos se empleó el método de Agrupación Pareado no Ponderados con Media Aritmética (Unweighted Pair Group Method using Arithmetic averages - UPGMA, Sokal y Michener 1958). El análisis se tuvo en cuenta para las especies, así como también para las parcelas y las estaciones del año. Este método calcula el promedio de grupos de pares sin ponderar, los clústeres se unen de acuerdo a la distancia media que existe entre todos los miembros de los dos grupos (Hammer 2016).

Para el análisis de las variables independientes (año, época del año, parcela) y dependientes (abundancias de las especies) se realizó un ANOVA y posteriormente un MANOVA, para ver si los cambios en las variables independientes tienen efectos significativos en las dependientes. El ANOVA factorial ayuda a evaluar el efecto de dos o más variables independientes sobre una variable dependiente. El análisis de MANOVA analiza la relación entre dos o más variables independientes (Hernández Sampieri et al. 2010) y puede demostrar asociaciones significativas no indicadas por los análisis univariados (Willig et al. 1986, Willig y Owen 1987).

Estos análisis multivariados ayudan a comparar y visualizar los efectos que el año, la parcela, o la época del año tienen en las abundancias de las especies de micromamíferos y en cuáles especies. Al visualizar al menos una diferencia entre las medias de los datos, es decir al rechazarse la hipótesis nula, se procedió con pruebas a posteriori de Tukey para saber cuáles medias fueron significativamente diferentes.

La prueba de normalidad de los datos, así como las pruebas de Análisis de la Varianza (ANOVA), Análisis Multivariado (MANOVA) y Análisis de Agrupamiento (clúster) se realizaron en el Programa Infostat (Di Rienzo et al. 2008). El análisis de Correspondencia Canónica (CCA) se realizó en el Programa Past (Hammer 2016).

\section{Resultados}

En total se registraron 16 especies de micromamíferos no voladores encontrados en los sitios de muestreo, con 1.161

Tabla 2. Abundancias (número mínimo conocido en vivo) para cada especie en cada cuadrícula durante cada sesión de muestreo.

\begin{tabular}{|c|c|c|c|c|c|c|c|c|c|c|c|c|c|}
\hline \multirow[b]{3}{*}{ Taxón/Especies } & \multicolumn{4}{|c|}{ Parcela B (Baja) } & \multicolumn{4}{|c|}{ Parcela A (Media) } & \multicolumn{4}{|c|}{ Parcela G (Alta) } & \multirow[b]{3}{*}{ Total } \\
\hline & \multicolumn{2}{|c|}{2015} & \multicolumn{2}{|c|}{2016} & \multicolumn{2}{|c|}{2015} & \multicolumn{2}{|c|}{2016} & \multicolumn{2}{|c|}{2015} & \multicolumn{2}{|c|}{2016} & \\
\hline & Jun & Nov & Jun & Nov & Jun & Nov & Jun & Nov & Jun & Nov & Jun & Nov & \\
\hline \multicolumn{14}{|l|}{ Didelphidae } \\
\hline Cryptonanus chacoensis & 0 & 0 & 0 & 0 & 0 & 1 & 0 & 0 & 0 & 0 & 0 & 0 & 1 \\
\hline Gracilinanus agilis & 5 & 0 & 4 & 0 & 1 & 1 & 2 & 4 & 3 & 2 & 6 & 1 & 29 \\
\hline Marmosa paraguayana & 1 & 0 & 0 & 0 & 0 & 0 & 0 & 0 & 0 & 0 & 0 & 0 & 1 \\
\hline Monodelphis dimidiata & 0 & 0 & 0 & 1 & 0 & 0 & 0 & 0 & 0 & 0 & 0 & 0 & 1 \\
\hline \multicolumn{14}{|l|}{ Cricetidae } \\
\hline Akodon montensis & 53 & 45 & 23 & 12 & 59 & 59 & 28 & 18 & 29 & 28 & 16 & 12 & 382 \\
\hline Calomys callosus & 0 & 1 & 0 & 0 & 1 & 0 & 0 & 0 & 4 & 3 & 0 & 0 & 9 \\
\hline Cerradomys maracujensis & 0 & 0 & 1 & 0 & 0 & 0 & 0 & 0 & 0 & 0 & 0 & 0 & 1 \\
\hline Hylaeamys megacephalus & 15 & 4 & 3 & 1 & 8 & 6 & 12 & 6 & 14 & 7 & 7 & 3 & 86 \\
\hline Juliomys pictipes & 0 & 0 & 1 & 0 & 0 & 0 & 0 & 0 & 0 & 0 & 0 & 0 & 1 \\
\hline Necromys lasiurus & 0 & 0 & 0 & 0 & 2 & 1 & 0 & 0 & 0 & 0 & 0 & 0 & 3 \\
\hline Oecomys cf. mamorae & 0 & 0 & 0 & 0 & 0 & 0 & 0 & 1 & 0 & 0 & 0 & 0 & 1 \\
\hline Oligoryzomys mattogrossae & 3 & 0 & 3 & 0 & 3 & 1 & 2 & 0 & 1 & 0 & 3 & 0 & 16 \\
\hline Oligoryzomys nigripes & 7 & 5 & 4 & 0 & 7 & 5 & 6 & 1 & 4 & 4 & 3 & 4 & 50 \\
\hline Oligoryzomys sp. & 0 & 0 & 0 & 0 & 4 & 0 & 2 & 0 & 1 & 0 & 2 & 0 & 9 \\
\hline Rhipidomys macrurus & 0 & 0 & 1 & 0 & 0 & 0 & 0 & 0 & 0 & 0 & 0 & 0 & 1 \\
\hline Sooretamys angouya & 0 & 0 & 0 & 2 & 0 & 1 & 0 & 0 & 0 & 0 & 1 & 3 & 7 \\
\hline Total & 84 & 55 & 40 & 16 & 85 & 75 & 52 & 30 & 56 & 44 & 38 & 23 & 598 \\
\hline
\end{tabular}


capturas de 598 individuos y 563 recapturas (Tabla 2). Del total, doce especies fueron del orden Rodentia (todos de la familia Cricetidae, subfamilia Sigmodontinae), y cuatro del orden Didelphimorphia (familia Didelphidae). Barreto Cáceres (2017) proporcionó los datos, y detalló los niveles de la riqueza y diversidad de especies, así como la acumulación de especies a través de tiempo.

Análisis de Correspondencias Canónicas. Para el análisis de correspondencias canónicas (CCA) con las características de la vegetación en cada sitio asociado a los distintos niveles de degradación, las variables medidas distancia al árbol más cercano (DAC) y árbol caído ( $\mathrm{AC}$ ), están relacionados positivamente a degradación media y tiene relación negativa con el hábitat de degradación alta. Las variables altura máxima del dosel (AMD) y madera muerta (MM) son asociadas con degradación baja. Árbol de naranjo (AN) y el porcentaje de cobertura de hierba $(\mathrm{PCH})$ se observó mayormente asociado a hábitats con alto nivel de degradación (vectores de hábitats no mostrados).

Las especies Akodon montensis (Am) y Oecomys cf. mamorae (Oem) se asociaron a las variables de distancia al árbol más cercano (DAC) y árbol caído (AC), relacionados con el hábitat de degradación media. Las especies Cerradomys maracajuensis $(\mathrm{Cm})$, Rhipidomys macrurus $(\mathrm{Rm})$ y Juliomys pictipes $(\mathrm{Jp})$ se encontraron influenciadas por la presencia de madera muerta (MM) en el hábitat con degradación baja. Mientras que la distribución de las especies Hylaeamys megacephalus (Hm), Necromys lasiurus (NI), Oligoryzomys mattogrossae (Om), Oligoryzomys sp. (Osp), Calomys callosus (Cc) junto con Gracilinanus agilis (Ga) Cryptonanus chacoensis (Cch) y Marmosa paraguayana (Mp) estuvo asociada al hábitat con degradación alta e influenciada por la presencia de árbol de naranjo (AN). Las especies Sooretamys angouya (Sa) Monodelphis dimidiata (Md) aparentemente no presentaron alguna relación estrecha con las variables mencionadas (Figura 1). En el análisis de los eigenvectores del análisis de correspondencia canónica, se observa que la mayor parte del análisis es explicada por el

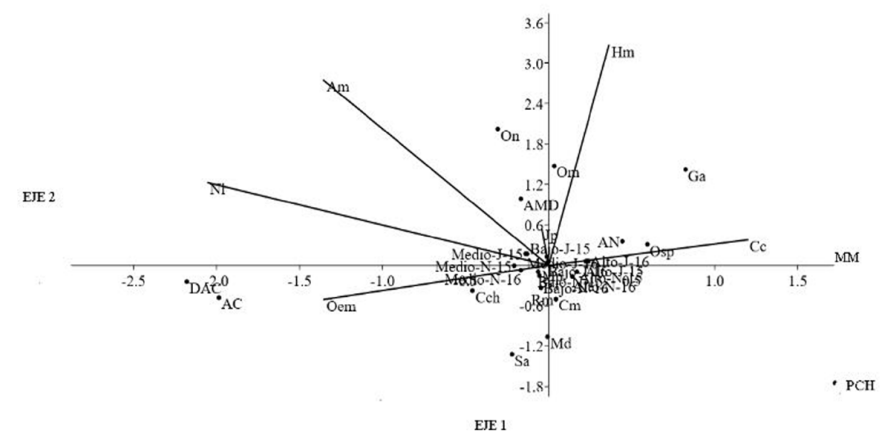

Figura 1. Análisis de Correspondencias Canónica (CCA) para las especies de micromamíferos y los sitios de estudio y las variables de vegetación. Referencias. Especies: Akodon montensis (Am), Calomys callosus (Cc), Cerradomys maracujensis (Cm), Cryptonanus chacoensis (Cch), Gracilinanus agilis (Ga), Hylaeamys megacephalus (Hm), Juliomys pictipes (Jp), Marmosa paraguayana (Mp), Monodelphis dimidiata (Md), Necromys lasiurus (NI), Oecomys cf. mamorae (Oem), Oligoryzomys mattogrossae (Om), Oligoryzomys nigripes (On), Oligoryzomys sp (Osp), Rhipidomys macrurus (Rm), Sooretamys angouya (Sa). Variables: Árbol caído (AC), Distancia al árbol más cercano (DAC), Porcentaje de cobertura de hierbas (PCH), Madera muerta (MM), Árbol de Naranjo (AN), Altura máxima del dosel (AMD). primer eje que aporta el $46.67 \%$ de la varianza y el segundo eje que aporta el $33.88 \%$ (Tabla 3).

Agrupamiento de especies. La Figura 2 muestra para la composición de especies para todos los sitios y épocas de muestreos. El grupo de las especies Akodon montensis (Am) y Hylaeamys megacephalus $(\mathrm{Hm})$ con Oligoryzomys nigripes (On) y Gracilinanus agilis (Ga) forman un nudo con la especie Oligoryzomys mattogrossae $(\mathrm{Om})$ que se separa de los demás grupos. Las especies Juliomys pictipes (Jp), Rhipidomys macrurus (Rm) y Cerradomys maracujensis (Cm), conforman otro grupo junto con las especies Oecomys cf. mamorae (Oem), Marmosa paraguayana (Mp) y Monodelphis dimidiata (Md), y juntos se encuentran conformando un punto de unión con las especies Necromys lasiurus (NI) y Cryptonanus chacoensis (Cch). La especie Sooretamys angouya ( $\mathrm{Sa}$ ) se une a los grupos anteriores. Las especies Calomys callosus (Cc) y Oligoryzomys sp. (Osp) se encuentran conformando otro grupo.

Agrupamiento de sitios, épocas y años. En la asociación con los tipos de hábitats y época del año, se relacionaron el grupo de los sitios de degradación alta y baja de la época lluviosa del 2016. Estos grupos se separan de los otros conformado por el hábitat con degradación alta y media de la época seca y lluviosa respectivamente del año 2016, conectados a su vez con el hábitat de degradación baja de la época seca del mismo año. Los otros tipos de hábitats que son degradación media y degradación baja de la época seca del año 2016 y 2015, respectivamente, se encuentran asociados al hábitat de degradación media de la época seca del 2015, y este a la vez con los hábitats de degradación alta de la época seca y lluviosa del 2015, relacionados con el hábitat de degradación media de la época lluviosa del 2015. Todos estos grupos se encuentran asociados formando un grupo con el hábitat de degradación baja de la época lluviosa del año 2015 (Figura 3).

Análisis de la varianza (ANOVA). En el ANOVA las variables analizadas fueron época del año, parcela y año. El análisis muestra que cinco especies presentaron diferencias significativas: Akodon montensis (año, parcela), Calomys callosus y Oligoryzomys nigripes (año), y Oligoryzomys mattogrossae y Hylaeamys megacephalus (época del año). Al realizar la prueba a posteriori de Tukey, para Akodon montensis las variables que demostraron ser significativas fueron año y parcela. La diferencia entre las parcelas se debe a que en el sitio con degradación alta $(G)$ se registró un número menor de individuos y en el sitio con degradación media (A) se observó un número mucho mayor de individuos (Tabla

Tabla 3. Componentes y valores de los porcentajes de varianza del análisis de CCA.

\begin{tabular}{|c|c|c|}
\hline Axis & Eigenvalue & $\%$ \\
\hline 1 & 0.15995 & 46.67 \\
\hline 2 & 0.11613 & 33.88 \\
\hline 3 & 0.04223 & 12.32 \\
\hline 4 & 0.01588 & 4.63 \\
\hline 5 & 0.00856 & 2.49 \\
\hline 6 & 0.00000 & 0.00 \\
\hline
\end{tabular}




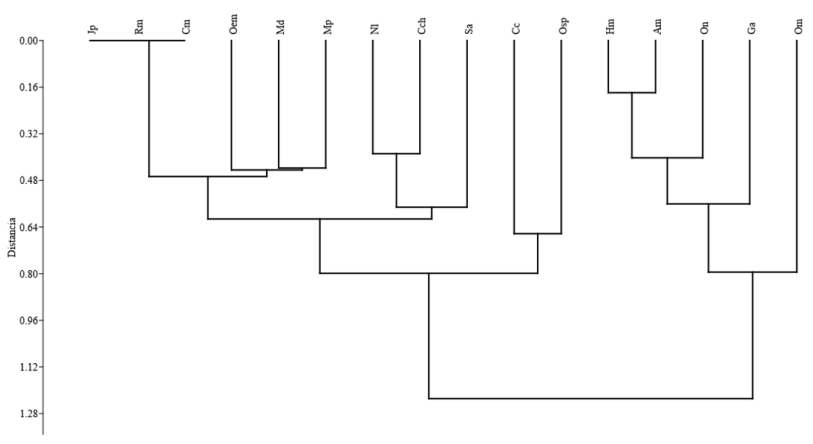

Figura 2. Análisis de agrupamiento para la composición de especies en todos los sitios, época y año que se realizaron los muestreos. Akodon montensis (Am), Calomys callosus (Cc), Cerradomys maracujensis (Cm), Cryptonanus chacoensis (Cch), Gracilinanus agilis (Ga), Hylaeamys megacephalus (Hm), Juliomys pictipes (Jp), Marmosa paraguayana (Mp), Monodelphis dimidiata (Md), Necromys lasiurus (NI), Oecomys cf. mamorae (Oem), Oligoryzomys mattogrossae (Om), Oligoryzomys nigripes (On), Oligoryzomys sp. (Osp), Rhipidomys macrurus (Rm), Sooretamys angouya (Sa).

4). Se rechaza así la hipótesis nula de igualdad de medias de todas las variables; por lo mismo, se acepta la hipótesis alternativa en la cual se indica que al menos una variable produce una influencia sobre la especie.

Para Hylaeamys megacephalus la variable que resultó significativa fue la época del año. La diferencia se debió a un número mayor en la época seca (junio) y menor en la época lluviosa (noviembre; Tabla 4). Sin embargo, en el modelo estadístico no resultó significativo $(P=0,096)$.

Para Calomys callosus lo que resultó significativo fue la variable año. Esto se debe a que no se encontró ningún ejemplar en el 2016, por eso resultó significativo para esa especie en el análisis (Tabla 4). Sin embargo, en el modelo estadístico no resultó significativo $(P=0,095)$.

Para Oligoryzomys nigripes también resultó significativa la variable año. Esto se debe a que se presentó un menor número en el 2016 (Tabla 4). Sin embargo, en el modelo estadístico no resultó significativo $(P=0,112)$.

Para Oligoryzomys mattogrossae lo que resultó significativo fue la variable época del año. Esto se debe a que solamente un individuo se encontró en la época lluviosa (Tabla 4). Sin embargo, en el modelo estadístico no resultó significativo $(P=0,009)$.

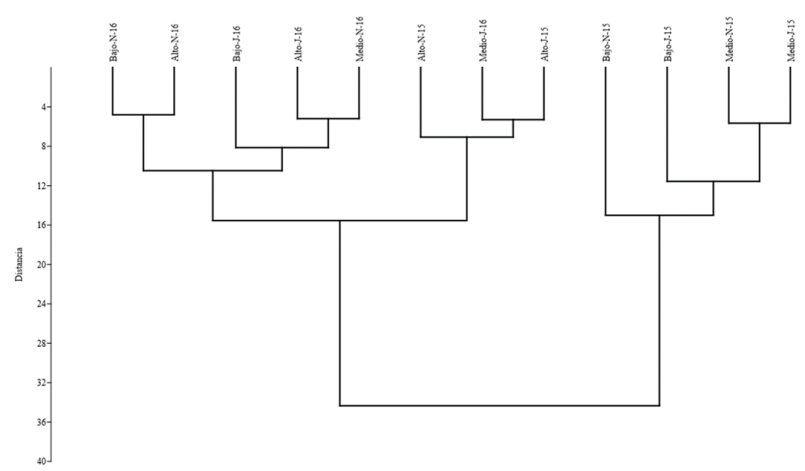

Figura 3. Análisis de agrupamiento para la composición de especies entre todos los sitios, época y año que se realizaron los muestreos. Alto-J-15 (nivel de degradación alto, junio de 2015), Alto-N-15 (nivel de degradación alto, noviembre de 2015), Alto-J-16 (nivel de degradación alto, junio de 2016), Alto-N-16 (nivel de degradación alto, noviembre de 2016), Bajo-J-15 (nivel de degradación bajo, junio de 2015), Bajo-N-15 (nivel de degradación bajo, noviembre de 2015), Bajo-J-16 (nivel de degradación bajo, junio de 2016), Bajo-N-16 (nivel de degradación bajo, noviembre de 2016), Medio-J-15 (nivel de degradación medio, junio de 2015), Medio-N-15 (nivel de degradación medio, noviembre de 2015), Medio-J-16 (nivel de degradación medio, junio de 2016), Medio-N-16 (nivel de degradación medio, noviembre de 2016).

Análisis de la varianza multivariado (MANOVA). El análisis de la varianza multivariado demostró que el año fue el patrón más significativo para la especie Akodon montensis. En el estadístico de Wilks se observan valores de la aproximación $F 2,16 ; 50,31$ y 8,89 para las variables época del año, año y parcela respectivamente (Tabla 5 ).

\section{Discusión}

Especies comunes. En Paraguay oriental, Akodon montensis es una especie común encontrada en los bosques, así también lo es por ejemplo en la zona de Misiones, Argentina, donde es más frecuente en diversos ambientes, sin incluir los bordes de arroyos (Cirignoli et al. 2011, de la Sancha 2014). En el presente estudio se observó que $A$. montensis estaba asociado a hábitats de degradación media en la época seca. En dicho sitio, estaban presentes comunidades vegetales de árboles de gran porte, hasta $25 \mathrm{~m}$ de altura, arbustos hasta $3 \mathrm{~m}$ de altura y con predominio de gramíneas en el sotobosque.

Lima et al. (2010) registraron una correlación positiva entre el número de capturas de $A$. montensis con la abun-

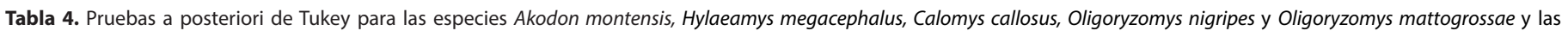

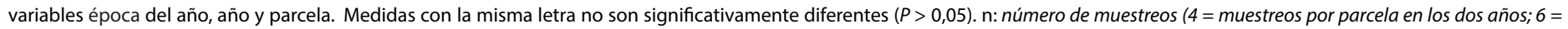
muestreos por época del año y año en los dos años).

\begin{tabular}{|c|c|c|c|c|c|c|c|c|c|c|c|c|c|c|c|c|}
\hline \multirow[b]{2}{*}{ Efecto } & \multicolumn{4}{|c|}{ Akodon montensis } & \multicolumn{3}{|c|}{ Hylaeamys megacephalus } & \multicolumn{3}{|c|}{ Calomys callosus } & \multicolumn{3}{|c|}{ Oligoryzomys nigripes } & \multicolumn{3}{|c|}{ Oligoryzomys mattogrossae } \\
\hline & Medias & $n$ & Grupo & & Medias & $n$ & Grupos & Medias & $n$ & Grupos & Medias & $n$ & Grupos & Medias & $n$ & Grupos \\
\hline \multicolumn{17}{|l|}{ Época del año } \\
\hline Nov. & 29.00 & 6 & & A & 4.50 & 6 & A & 0.67 & 6 & A & 3.17 & 6 & A & 0.17 & 6 & A \\
\hline Jun. & 34.67 & 6 & & A & 9.83 & 6 & B & 0.83 & 6 & A & 5.17 & 6 & A & 2.50 & 6 & B \\
\hline \multicolumn{17}{|l|}{ Año } \\
\hline 2016 & 18.17 & 6 & & A & 5.33 & 6 & A & 0.00 & 6 & A & 3.00 & 6 & A & 1.33 & 6 & A \\
\hline 2015 & 45.50 & 6 & & B & 9.00 & 6 & A & 1.50 & 6 & B & 5.33 & 6 & B & 1.33 & 6 & A \\
\hline \multicolumn{17}{|l|}{ Parcela } \\
\hline G & 21.25 & 4 & & A & 7.75 & 4 & A & 1.75 & 4 & A & 3.75 & 4 & A & 1.00 & 4 & A \\
\hline B & 33.25 & 4 & $A$ & B & 5.75 & 4 & A & 0.25 & 4 & A & 4.00 & 4 & A & 1.50 & 4 & A \\
\hline A & 41.00 & 4 & & B & 8.00 & 4 & A & 0.25 & 4 & A & 4.75 & 4 & A & 1.50 & 4 & A \\
\hline
\end{tabular}


dancia de bambú, pudiendo estar asociado a la protección contra los depredadores. En contraste, Goodin et al. (2009) indican que $A$. montensis es conocido por ser generalista en cuanto al hábitat, pero muestra preferencia por áreas boscosas semicaducifolias, donde los estratos superiores son menos densos y la cobertura vegetal más densa cerca del suelo. La mayor abundancia de $A$. montensis se registró en los microhábitats que mostraban un grado de actividad antropogénica con mayor penetración de luz, pero donde el bosque nativo estaba aun sustancialmente intacto. Las áreas con vegetación densa y la superficie del suelo desnuda o con poca vegetación tenían menos probabilidades de estar asociadas con la presencia de A. montensis (Goodin et al. 2009). Melo et al. (2013), observaron una relación positiva entre $A$. montensis con los helechos y troncos caídos en el Bosque Atlántico del estado de Rio Grande do Sul, Brasil. Püttker et al. (2008) señalan a A. montensis como una especie no vulnerable a la fragmentación de los bosques. Las variables significativas en este estudio para A. montensis fueron la variable año y la variable parcela o hábitat. Esto se debe a que en el 2016 el número de ejemplares de esta

Tabla 5. Análisis de la varianza multivariado, para la especie Akodon montensis. Esta especie resultó significativa en el modelo estadístico de ANOVA. Cuadro de Análisis de la Varianza (Wilks). Obsérvese el $p<0.05$ para la variable año $(p=0.0002)$ y parcela $(p=$ $0.0120)$, ambas variables fueron significativas. Sin embargo, la variable año influenció en mayor medida en la población de la especie. gl: grados de libertad

\begin{tabular}{lccccc}
\hline \multicolumn{1}{c}{ Efecto } & Estadístico & \multicolumn{1}{c}{ F } & gl (num) & gl (den) & $P$ \\
\hline Época del año & 0.76 & 2.16 & 1 & 7 & 0.1849 \\
Año & 0.12 & 50.31 & 1 & 7 & 0.0002 \\
Parcela & 0.28 & 8.89 & 2 & 7 & 0.0120 \\
\hline
\end{tabular}

especie fue menor. Además, se podría inferir en una preferencia del hábitat (con nivel de degradación media) para esta especie.

La especie segunda más común fue Hylaeamys megacephalus, que no demostró una preferencia en el hábitat y que podría ser generalista, ya que se encuentra en los bosques primarios, secundarios y degradados (Percequillo et al. 2016). Se observó una variabilidad a nivel estacional, que sin embargo en el modelo estadístico no resultó significativa. En la época seca se encontró mayor registro de esta especie, en contraste a la disminución de la población en noviembre durante la época lluviosa. En las zonas boscosas y el cerrado de la parte central de Brasil, H. megacephalus se encontró solamente en la época húmeda (Santos-Filho et al. 2012). Sin embargo, en este estudio la población de $H$. megacephalus disminuyó en la época lluviosa. Se observó mayor abundancia de esta especie en la época seca (junio) y menor en la época lluviosa (noviembre).

Para la especie Oligoryzomys nigripes ninguna de las variables resultó significativa. Sin embargo, estuvo presente en los cuatro periodos de muestreo del estudio excepto en la época lluviosa del 2016. En la época seca de los dos años, mostró asociación con el sitio de degradación media, que podría deberse a características de la vegetación en esa época. Sponchiado et al. (2012) encontraron una relación positiva entre $O$. nigripes y las bromelias terrestres, junto con la cercanía a cuerpos de agua. Esta asociación no se observó en este estudio, ya que el predominio de bromelias se encontraba en el sitio de degradación alta. Esta es una de las especies menos especialistas en cuanto a la alimentación, por lo tanto, podría ser generalista en lo referente al hábitat. En la época seca, prefiere sitios más abiertos, también con algunos arbustos y árboles (Bonvicino et al. 2016). Se registró la presencia de $O$. nigripes asociado al dosel bajo y a un sotobosque denso en el Bosque Atlántico de Brasil, en zonas con vegetación característica de un bosque perturbado (Püttker et al. 2008; Lima et al. 2010). Se considera a $O$. nigripes como una especie no vulnerable a la fragmentación de los bosques (Püttker et al. 2008). Galiano et al. (2013) encontraron que la dominancia poblacional entre $A$. montensis y $O$. nigripes alternó en un Bosque de Araucaria, en el sur de Brasil. La abundancia de A. montensis no correspondió a ningún factor extrínseco (precipitación, temperatura, etc.), mientras la de 0 . nigripes aumentó en el invierno. Eastwood et al. (2018) determinaron que las especies $A$. montensis y $O$. nigripes son simpátricas en la RNBM, en el que fue el primer estudio para determinar los hábitats naturales de dos especies simpátricas de roedores.

En cuanto a los marsupiales, Gracilinanus agilis resultó más común dentro del grupo de los didélfidos. Sin embargo, sus poblaciones se mostraron muy variables a través del tiempo y entre sitios. En Mato Grosso do Sul, Brasil se observó que la población de G. agilis presentó fluctuación marcadamente estacional, con una alta concentración de la especie en la época lluviosa. Se presume que G. agilis tiene tolerancia a algún grado de modificación del hábitat (Carmignotto et al. 2015). En esta investigación se observó que $G$. agilis fue fluctuando de igual manera durante todos los periodos de muestreos en las diferentes estaciones del año y asociado a los tres niveles de degradación.

Efectos de caracteres vegetales y degradación. Las asociaciones entre comunidades de micromamíferos y comunidades vegetales, indicarían preferencia hacia los diferentes tipos de microhábitats por parte de los micromamíferos. Estas preferencias podrían ser un factor considerable que influye en los pequeños mamíferos para habitar zonas que pueden estar alteradas (Püttker et al. 2008; Lima et al. 2010). Sin embargo, el estado de conservación de las especies de micromamíferos de la zona de estudio se encuentra dentro de la categoría de Preocupación Menor (LC) según la lista roja de la UICN (2009). Esto podría deberse a que se reproducen rápidamente y tienen un ciclo de vida relativamente corto. Sin embargo, la alteración de los bosques y hábitats de pequeños mamíferos podría poner en riesgo a las especies más sensibles, sobre todo de marsupiales. Cabe destacar que tres especies (Marmosa paraguayana, Delomys dorsalis y Juliomys pictipes) que se encuentran en la Reserva Natural del Bosque Mbaracayú son endémicas del BAAPA (Owen y Smith 2019). En este estudio se registraron a Marmosa paraguayana y Juliomys pictipes. 
Las comunidades vegetales son principalmente importantes para las comunidades de micromamíferos que se encuentran interaccionando en los diferentes hábitats del bosque. La alteración o degradación del bosque puede llegar a intervenir con el desequilibrio temporal de las poblaciones y comunidades de pequeños mamíferos (Pardini y Umetsu 2006). Las especies consideradas no vulnerables a la fragmentación se asocian a zonas con características de la vegetación de un bosque más perturbado. En cambio, las especies vulnerables a la fragmentación se encontrarían en áreas con características de los bosques primarios (Püttker et al. 2008). Estos patrones se observaron en este estudio ya que se encontraron especies más asociadas a áreas perturbadas y otras especies en zonas de degradación baja. De la Sancha (2014) encontró patrones similares en la Reserva Natural del Bosque Mbaracayú con los bosques megadiversos como el Amazonas. Sin embargo, a pesar de ser uno de los fragmentos de bosques más grandes del BAAPA, Mbaracayú mostró números considerablemente bajos de muestra para pequeños mamíferos comparando con otros fragmentos. Asimismo, en la Amazonía peruana se llegó a reportar números de capturas extremadamente bajas para micromamíferos no voladores, es decir en bosques vírgenes (Hice y Velazco 2012; de la Sancha 2014). En este estudio también se registraron bajos números de muestras, sobre todo en marsupiales y algunos roedores, a pesar de que el esfuerzo de muestreo invertido fue considerable. Sin embargo, podría ser un patrón seguido para los bosques de grandes fragmentos como el Bosque del Mbaracayú.

Efectos de época del año. Para las especies Hylaeamys megacephalus y Oligoryzomys mattogrossae solo la época del año resultó significativa en el análisis de la varianza. Sin embargo, el modelo estadístico para éste análisis no demostró significancia alguna. Este patrón asociado a una de las especies más abundantes como $\mathrm{H}$. megacephalus, sugiere que la época del año (seca y lluviosa) es un factor de importancia a tener en cuenta para las zonas del Bosque Atlántico. Esta variable no resultó significativa para las especies más comunes de roedor (Akodon montensis) y de didélfido (Gracilinanus agilis). En un estudio realizado en la zona del Cerrado en Brasil, Carmignotto et al. (2014) observaron mayor abundancia para la especie $H$. megacephalus en la época lluviosa que en la época seca, presumiendo una mayor actividad en la primera por la disponibilidad de alimentos. En contraste en esta investigación se observaron más individuos en la época seca, pudiendo deberse a que en la zona boscosa se encuentra una disponibilidad de recursos igualmente en la época seca. Para O. mattogrossae se podría deber a que se encontró solo un ejemplar en la época lluviosa del 2015, posiblemente debido a el fenómeno de El Niño, que será tratado más adelante. En una zona centro de Brasil la abundancia de micromamíferos resultó alta en la temporada seca y baja en la temporada húmeda (Santos-Filho et al. 2012). Así mismo, en este estudio se encontraron más especies de micromamíferos en la época seca que en la época lluviosa. Es importante conocer cómo influye un factor puntual como la época del año, ya que ayudaría a predecir la dinámica de las poblaciones de micromamíferos, sobre todo de las especies que encontramos más abundantes.

En una zona de la Mata Atlántica de Brasil encontraron que el promedio de especies de micromamíferos obtenidas por sitio de muestreo al término de la época lluviosa prácticamente duplicó lo que se obtuvo al final de la época seca (Pardini y Umetsu 2006), en contraste a este estudio donde se observó que en la época lluviosa disminuyen las abundancias.

Efectos del año. La variable año resultó significativa para la especie Akodon montensis en el análisis de la varianza multivariado. Los valores bajos de $p$ para esta variable sugieren que las abundancias de esta especie cambian de acuerdo a la época del año y más aún entre años. El año 2015 fue un año de altas poblaciones para A. montensis. Sin embargo, la abundancia de roedores disminuyó en el año 2016, y algunas especies de marsupiales sólo estuvieron presente en un año de estudio, lo que podría deberse a las constantes precipitaciones que se dieron a finales del 2015, cuando la región experimentó un evento extremo de El Niño (Dirección Nacional de Aeronáutica Civil 2016). Este fenómeno pudo incidir en algunas características o cambios de la vegetación y por ende en la preferencia o presencia o no de las especies a los sitios muestreados (Owen 2013). Para Calomys callosus y Oligoryzomys nigripes solo el año resultó significativo en el análisis de la varianza. Sin embargo, el modelo estadístico para este análisis no demostró significancia. Esto se debe a que la especie C. callosus se encontró solo en el 2015 y no hubo alguna asociación con el siguiente año de estudio. Su ocurrencia en un solo año podría deberse a que es considerada una especie rara en este estudio ya que los patrones de distribución de esta especie no son considerados para esta ecorregión (de la Sancha 2014). En el caso de O. nigripes podría deberse a su abundancia mayor en el año 2015, relacionado posiblemente al efecto de El Niño.

\section{Conclusiones}

El sitio con mayor riqueza fue el de degradación media, en las estaciones seca y lluviosa del año 2015 y el de degradación baja en la época seca del año 2016. En el 2016 se registró la menor cantidad de especies en la época lluviosa en el hábitat con degradación media y degradación alta, así como también en el hábitat con degradación baja y alta en la época lluviosa del 2015. Los miembros de la familia Cricetidae fueron los más numerosos en todo el muestreo, en donde se encuentran Akodon montensis y Hylaeamys megacephalus. Para la familia Didelphidae, la especie con más ocurrencia fue Gracilinanus agilis, encontrado en todos los hábitats.

Las especies Akodon montensis (Am), Oligoryzomys nigripes (On) así como Oecomys cf. mamorae (Oem) se asociaron más a la distancia al árbol más cercano (DAC) y árbol 
caído $(A C)$, relacionados con el hábitat de degradación media. Las especies Cerradomys maracujensis (Cm), Rhipidomys macrurus $(\mathrm{Rm})$ y Juliomys pictipes $(\mathrm{Jp})$ se encontraron influenciadas por la presencia de madera muerta (MM) en el hábitat con degradación baja. Mientras que la distribución de las especies Hylaeamys megacephalus (Hm), Necromys lasiurus (NI) Oligoryzomys mattogrossae (Om), Oligoryzomys sp. (Osp), Calomys callosus (Cc) junto con Gracilinanus agilis (Ga) Cryptonanus chacoensis (Cch) y Marmosa paraguayana (Mp) estuvo asociada al hábitat con degradación alta e influenciada por la presencia deárbol de naranjo (AN)..

El sitio con degradación media fue el sitio con más riqueza en el 2015. Sin embargo, hubo una variación en el siguiente año siendo uno de los sitios con menos especies. La variable año resultó significativa para Akodon montensis, ya que hubo un descenso en la población de estas especies en el 2016. Las distintas especies (sobre todo las más abundantes) responden de diferentes maneras a las modificaciones del hábitat (variable parcela) y a lo largo del ciclo anual y en las diferentes épocas debido a sus preferencias u ocurrencia en el hábitat. La mayoría de las especies que no fueron muy abundantes serían más específicas de cada sitio o estructura de la vegetación. Al igual que la época del año, la diferencia que marcaron los dos años de estudio es un patrón que predice el comportamiento de las poblaciones de micromamíferos en ese periodo de tiempo. Esto ayuda a visualizar por ejemplo cómo cambian las poblaciones de micromamíferos no voladores a lo largo de un año.

Se recomienda estudios longitudinales para observar la dinámica de la población, observar las respuestas de las especies poco comunes y de las especies de roedores y marsupiales en general.

\section{Agradecimientos}

A la Texas Tech University, por la oportunidad brindada a través de la beca ofrecida para la realización de esta investigación y al National Institute of Health (NIH, EE.UU.) (I103053) y NIH R01 TW006986-01 a través del Programa de Ecología de Enfermedades Infecciosas de NIH-NSF. A los Profesores de la Coordinación de Postgrado, K. Núñez, A. Weiler, G. González, por el excelente trabajo llevado a cabo en todo este periodo. En especial a F. Silla por la valiosa ayuda brindada. A la Fundación Moisés Bertoni, en especial a las personas de la Reserva Natural del Bosque Mbaracayú, por ser tan buenos anfitriones y personas admirables. A las personas que nos ayudaron en el arduo trabajo de campo, E. Galeano, V. Martínez, H. Sánchez, J. Sánchez, E. Ríos, A. Alfonzo, M. Sánchez, A. Rivarola y D. Bueno. A G. D’Elía e I. Gamarra, que con sus comentarios y consejos contribuyeron en diferentes aspectos de este trabajo. A los revisores del artículo por las sugerencias aportadas para el enriquecimiento del trabajo.

\section{Literatura citada}

Aragón, E. E., A. Garza, y F. A. Cervantes. 2009. Estructura y organización de los ensambles de roedores de un bosque de la Sierra Madre occidental, Durango, México. Revista Chilena de Historia Natural 82:523-542.

Barreto Cáceres, M. B. 2017. Relación de los micromamíferos no voladores (Rodentia y Didelphimorphia) con la estructura de la vegetación y el grado de perturbación del bosque en una zona del límite occidental del Bosque Atlántico del Alto Paraná. M. C. tesis, Universidad Nacional de Asunción, San Lorenzo, Paraguay.

Bonaventura, S. M., V. Pancotto, N. Madanes, y R. Vicari. 2003. Microhabitat use and density of sigmodontine rodents in Spartina densiflora fresh water marshes, Argentina. Mammalia 67:367-377.

Bonvicino, C., G. D'eliA, y P. Teta. 2016. Oligoryzomys nigripes. In the IUCN Red List of Threatened Species. Version 2016. www. iucnredlist.org. Consultado el 22 de de abril de 2017.

Carmignotto, A. P., A. M. R. Bezerra, y F. H.G. Rodrigues. 2014. Nonvolant small mammals from a southwestern area of Brazilian Cerrado: diversity, habitat use, seasonality, and biogeography. Therya 5:535-558.

Carmignotto, A. P., S. Solari, N. de La Sancha, y L. Costa. 2015. Gracilinanus agilis. . In $\mathrm{t}$ he IUCN Red List of Threatened Species. Version 2015. www.iucnredlist.org. Consultado el 22 de de abril de 2017.

Cartes, J. (ed.). 2006. El Bosque Atlántico en Paraguay: Biodiversidad, Amenazas y Perspectivas. Asociación Guyrá Paraguay / Conservation Internacional Center for Applied Biodiversity Science. Asunción, Paraguay.

Cirignoli, S., C. Galliari, U. F. J. Pardiñas, D. H. Podestá, y R. Abramson. 2011. Mamíferos de la reserva Valle del Cuña Pirú, Misiones, Argentina. Mastozoología Neotropical 18:25-43.

DE LA SANCHA, N. U. 2014. Patterns of small mammal diversity in fragments of subtropical Interior Atlantic Forest in eastern Paraguay. Mammalia 78:437-449.

Delciellos, A. C., M. Vieira, C. E. V. Grelle, P. Cobra, y R. Ceroueira. 2016. Habitat quality versus spatial variables as determinants of small mammal assemblages in Atlantic Forest fragments. Journal of Mammalogy 97:253-265.

Dı BItettI, M. S., G. PlacCI, Y L. A. Dietz. 2003. A biodiversity vision for the Upper Parana Atlantic Forest ecoregion: designing a biodiversity conservation landscape and setting priorities for conservation action. World Wildlife Fund. Washington, EE.UU.

Dirección nacional de Aeronáutica Civil (DinaC). Dirección de Meteorología e Hidrología. 2016. El Episodio de El Niño 2015/16 Excepcionalmente intenso. Efectos en Paraguay. Gerencia de Climatología e Hidrología. Asunción, Paraguay.

Dinerstein, E., D. Olson, D. Graham, A. Webster, S. Primm, M. BOOKBINDER, Y G. LEDEC. 1995. Una evaluación del estado de conservación de las ecorregiones terrestres de América Latina y el Caribe. WWF, Banco Mundial. Washington, EE.UU. Di Rienzo, J. A., F. Casanoves, M. G. Balzarinı, L. Gonzalez, M. TAblada, y C. W. Robledo. 2008. InfoStat, versión 2008, Grupo InfoStat, FCA, Universidad Nacional de Córdoba. Córdoba, Argentina. Eastwood, G., J. V. CAmp, Y. K. Chu, A. M. SaWyer, R. D. OWen, X. CAo, M. K.Taylor, L. Valdivieso-Torres, R. D. Sage, A. Yu, D. G. Goodin, V. J. Martínez Bruyn, R. C. McAllister, L. Rodriguez, E. P. Williams, y C. B. Jonsson. 2018. Habitat, species richness and hantaviruses of sigmodontine rodents within the Interior Atlantic Forest, Paraguay. PloS ONE 13:1-20. 
Emmons, L. H. 1999. Mamíferos de los Bosques Húmedos de América Tropical. Fundación Amigos de la Naturaleza FAN. Santa Cruz de la Sierra, Bolivia.

FMB/BM. (eds). 2005. Reserva Natural del Bosque Mbaracayú. Plan de Manejo 2005-2010. Fundación Moisés Bertoni para la Conservación de Naturaleza (FMB). Banco Mundial (BM). Asunción, Paraguay.

Galiano, D., B. Busnelo Kubiak, J. Reppold Marinho, y T. R. Ochotorena De Freitas. 2013. Population dynamics of Akodon montensis and Oligoryzomys nigripes in an Araucaria forest of Southern Brazil. Mammalia 77:173-179.

GardneR, A. L. (ed). 2008. Mammals of South America, Marsupials, Xenarthrans, Shrews, and Bats. Volume 1. The University of Chicago Press. Chicago, EE.UU.

Goodin, D. G., R. Paige, R. D. Owen, K. Ghimire, D. E. Koch, Y-K. ChU, y C. B. Jonsson. 2009. Microhabitat characteristics of Akodon montensis, a reservoir for hantavirus, and hantaviral seroprevalence in an Atlantic forest site in eastern Paraguay. Journal of Vector Ecology 34:104-113.

Hammer, Ø. 2016. Paleontological Statistics. Version 3.14. Reference manual. Natural History Museum. University of Oslo. Oslo, Noruega.

Hernández Sampieri, R., C. Fernández, y M. Baptista. 2010. Metodología de la Investigación. Quinta edición. Mc Graw Hill. México, México.

HiCE, C. L., y P. M. VelazCo. 2012. The non-volant mammals of the Reserva Nacional Allpahuayo-Mishana, Loreto, Peru. Special Publications Museum of Texas Tech University. Texas Tech University. Lubbock, Texas:1-135.

Hickman, C., L. Roberts, y A. LARSON. 2002. Principios Integrales de Zoología. Undécima Edición. Interamericana McGraw Hill. Madrid, España.

JAKSIC, F., Y M. LIMA. 2003. Myths and facts on ratadas: Bamboo blooms, rainfall peaks and rodent outbreaks in South America. Austral Ecology 28:237-251

Lima, D. O. de, B. O. Azambuja, V. L. Camilotti, y N. C. Cáceres. 2010. Small mammal community structure and microhabitat use in the austral boundary of the Atlantic Forest, Brazil. Zoologia (Curitiba) 27:99-105.

Melo, G. L., J. Sponchiado, A. F. Machado, y N. C. Cáceres. 2011. Small-mammal community structure in a South American deciduous Atlantic Forest. Community Ecology 12:58-66.

Melo, G. L., B. Miotto, B. Peres, y N. C. Cáceres. 2013. Microhabitat of small mammals at ground and understorey levels in a deciduous, southern Atlantic Forest. Anais da Academia Brasileira de Ciências 85:727-736

Myers, N., R. A. Mittermeir, C. G. Mittermeir, G. A. G. de Fonseca y J. KENTS. 2000. Biodiversity hotspots for conservation priorities. Nature 403:853-858.

NAIDOO, R., y K. HILL. 2006. Emergence of indigenous vegetation classifications through integration of traditional ecological knowledge and remote sensing analyses. Environmental Management 38: 377-387.

OWEN, R. D. 2013. Ecology of small terrestrial mammals in an isolated Cerrado patch, eastern Paraguay: communities, species, and effects of ENSO, precipitation, and fire. Mastozoología Neotropical 20:97-112.

OWEn R. D., y P. SMith. 2019. Mamíferos de la Reserva Natural del Bosque Mbaracayú, Departamento Canindeyú
Representadas en Colecciones Científicas. Revista de la Sociedad Científica del Paraguay 24:163- 172.

Owen, R. D., D. G. Goodin, D. E. KoCH, Y-K. CHU, y C. B. Jonsson. 2010. Spatiotemporal variation in Akodon montensis (Cricetidae: Sigmodontinae) and hantaviral seroprevalence in a subtropical forest ecosystem. Journal of Mammalogy 91:467-481.

OWen, R. D., H. SÁnchez, L. Rodríguez, y C. B. Jonsson. 2018. Composition and characteristics of a diverse didelphid community (Mammalia: Didelphimorphia) in sub-tropical South America. Occasional Papers, Museum of Texas Tech University 358:1-18.

OWen, R. D., J. V. Camp, y C. B. Jonsson. 2019. Sigmodontine community and species responses to El Niño and precipitation in different levels of forest degradation. Therya 10:255-256.

PARDINI, R., Y F. UMETSU. 2006. Pequenos mamíferos nãovoadores da Reserva Florestal do Morro Grande-distribuição das espécies e da diversidade em uma área de Mata Atlântica. Biota Neotropica 6:1-22.

Patton, J., U. F. J. Pardiñas, y G. D'elía (eds.). 2015. Mammals of South America. Vol 2. Rodents. The University of Chicago Press. Chicago and London.

Peña-Chocarro, M. C., C. Espada-Mateos, M. Vera, G. Céspedes, y S. Knapp. 2010. Updated checklist of vascular plants of the Mbaracayú Forest Nature Reserve (Reserva Natural del Bosque Mbaracayú), Paraguay. Phytotaxa 12:1-224.

Percequillo, A., J. Patton, L. Pires-Costa, G. D’Elía, y B. Patterson. 2016. Hylaeamys megacephalus. En: IUCN 2016. The IUCN Red List of Threatened Species. Version 2016. www.iucnredlist. org. Consultado el 22 de de abril de 2017.

Poindexter, C. J., G. D. Schnell, C. Sánchez-Hernández, M. de L. Romero-Almaraz, M. L. Kennedy, T. L. Best, M. C. Wooten, y R. D. OWEN. 2012. Co-occurrence of small mammals in a tropical dry deciduous forest: comparisons of communities and individual species in Colima, Mexico. Journal of Tropical Ecology 28:65-72.

Polop, F., L. Sepúlveda, A. Pelliza Sbriller, J. Polop, y M. C. Provensal. 2014. Food habits of Oligoryzomys longicaudatus (Rodentia) in a steppe - forest transitional area of Argentinean Patagonia. Ecología Austral 24:304-310.

Püttker, T., R. PARdini, Y. Meyer-Lucht, y S. Sommer. 2008. Responses of five small mammal species to micro-scale variations in vegetation structure in secondary Atlantic Forest remnants, Brazil. BioMed Central Ecology 8:1-10.

Santos-Filho, M., F. Frieiro-Costa, I. Ára, y M. N. F. Silva. 2012. Use of habitats by non-volant small mammals in Cerrado in Central Brazil. Brazilian Journal of Biology 72:893-902.

Schnell, G. D., M. de L. Romero-Almaraz, S. T. Martínez-Chapital, C. Sánchez-Hernández, M. L. Kennedy, T. L. Best, M. C. Wooten, y R. D. OWEN. 2010. Habitat use and demographic characteristics of the west Mexican cotton rat (Sigmodon mascotensis). Mammalia 74:379-393.

Sikes, R. S., W. L. Gannon, y The Animal Care and Use Committee of the American Society of Mammalogists. 2011. Guidelines of the American Society of Mammalogists for the use of wild mammals in research. Journal of Mammalogy 92:235-253.

Sikes, R., y The Animal Care and Use Committee of the American Society of Mammalogists. 2016. Guidelines of the American Society of Mammalogists for the use of wild mammals in research and education. Journal of Mammalogy 97:663-688. 
Sneath, P. H., y R. R. Sokal. 1973. Numerical Taxonomy: The Principles and Practice of Numerical Classification. $1^{\text {st }}$ Edition, W. H. Freeman. San Francisco, EE.UU.

SOKAL, R. R., Y C. D. MiCHENER. 1958. A Statistical Methods for Evaluating Relationships. University of Kansas Science Bulletin 38:1409-1448.

Sponchiado, J., G. L. Melo, y N. C. CÁceres. 2012. Habitat selection by small mammals in Brazilian Pampas biome. Journal of Natural History 46:1321-1335.

Ter BraAk, C. J. F. 1986. Canonical correspondence analysis: a new eigenvector technique for multivariate direct gradient analysis. Ecology 67:1167-1179.

UICN. 2009. The UICN species survival Commission 2009 (en línea). The UICN Red List of Threatened Species. www. iucnredlist.org. Consultado abril 2017.

Voss, R., Y S. JANSA. 2009. Phylogenetic relationships and classification of didelphid marsupials, an extant radiation of New World metatherian mammals. Bulletin of The American Museum of Natural History 322:142-156.

WiLLI, M. R., R. D. Owen, y R. L. Colbert. 1986. Assessment of morphometric variation in natural populations: the inadequacy of the univariate approach. Systematic Zoology 35:195-203.

WILLIG, M. R., Y R. D. OWEN. 1987. Univariate analyses of morphometric variation do not emulate the results of multivariate analyses. Systematic Zoology 36:398-400.

Associated editor: Sergio Solari

Submitted: May 7, 2019; Reviewed: June 21, 2019;

Accepted:August 27, 2019; Published on line:September 25, 2019. 
370 THERYA Vol. 10 (3): 359-369 